Article

\title{
Excitons in Solids from Time-Dependent Density-Functional Theory: Assessing the Tamm-Dancoff Approximation
}

\author{
Young-Moo Byun and Carsten A. Ullrich * \\ Department of Physics and Astronomy, University of Missouri, Columbia, MO 65211, USA; \\ byuny@missouri.edu \\ * Correspondence: ullrichc@missouri.edu \\ Academic Editor: Jianmin Tao \\ Received: 21 December 2016; Accepted: 23 January 2017; Published: 29 January 2017
}

\begin{abstract}
Excitonic effects in solids can be calculated using the Bethe-Salpeter equation (BSE) or the Casida equation of time-dependent density-functional theory (TDDFT). In both methods, the Tamm-Dancoff approximation (TDA), which decouples excitations and de-excitations, is widely used to reduce computational cost. Here, we study the effect of the TDA on exciton binding energies of solids obtained from the Casida equation using long-range-corrected (LRC) exchange-correlation kernels. We find that the TDA underestimates TDDFT-LRC exciton binding energies of semiconductors slightly, but those of insulators significantly (i.e., by more than 100\%), and thus it is essential to solve the full Casida equation to describe strongly bound excitons. These findings are relevant in the ongoing search for accurate and efficient TDDFT approaches for excitons.
\end{abstract}

Keywords: excitons; time-dependent density-functional theory; Tamm-Dancoff approximation

\section{Introduction}

Excitons are bound electron-hole pairs arising in optically excited finite and extended systems. Understanding and predicting excitonic properties is important for the design of novel photovoltaic materials. For example, low exciton binding energies in perovskite solar cells promote the electron-hole separation and thereby enhance power conversion efficiencies [1].

Many-body perturbation theory is a standard method to calculate excitonic properties of solids: one obtains accurate exciton binding energies $E_{\mathrm{b}}$ and optical absorption spectra of semiconductors and insulators by solving the Bethe-Salpeter equation (BSE) [2]. However, the BSE is computationally expensive and cannot be applied to large systems.

Time-dependent density-functional theory (TDDFT) is a computationally cheaper alternative to the BSE [3], but its application to the study of excitonic effects in solids depends on finding good approximations to the unknown exchange-correlation (xc) kernel $f_{\mathrm{xc}}$. The random-phase approximation (RPA) (i.e., $f_{\mathrm{xc}}=0$ ), the local-density approximation (LDA), as well as any standard gradient-corrected semilocal approximation fail to capture excitonic properties of solids due to their inadequate long-range behavior. A very accurate xc kernel can be derived by reverse-engineering the BSE [2,4], but it is computationally as expensive. A drastic simplification, known as the long-range-corrected (LRC) kernel,

$$
f_{\mathrm{xC}}^{\mathrm{LRC}}=-\frac{\alpha}{\mathbf{q}^{2}},
$$

where $\mathbf{q}$ is a momentum transfer in the first Brillouin zone (BZ), accounts for bound excitons in solids, but it requires a material-dependent parameter $\alpha$, a positive scalar. Inspired by the simple form (1), a whole family of LRC-type kernels have been proposed in the literature [5-9]. 
The performance of LRC-type kernels is typically judged by how well they appear to reproduce experimental optical absorption spectra. The quality of a spectrum is usually assessed by inspection, and it depends strongly on the underlying band structure and on the numerical broadening. A better quantitative measure are exciton binding energies, which are defined as the energetic separation between the exciton and the band gap, and can hence be precisely quantified (to within experimental and numerical error bars). Furthermore, there is no numerical broadening in our scheme (see below), and the dependence on the choice of band structure is much weaker.

Experimentally, $E_{\mathrm{b}}$ can be directly extracted from the optical absorption spectra for the case of strongly bound excitons in insulators; for semiconductors, $E_{\mathrm{b}}$ can be obtained from photoluminescence data. The direct calculation of exciton binding energies can be achieved by solving the so-called Casida equation of TDDFT [10-12]. This approach is sometimes referred to as "diagonalizing the exciton Hamiltonian", and is formally similar in BSE and TDDFT. Usually, this is done within the Tamm-Dancoff approximation (TDA), which neglects the coupling between resonant and anti-resonant excitations. There are some recent studies investigating the performance of the TDA for the BSE [13,14]; however, the extent to which the TDA affects the solution of the excitonic Casida equation has not been studied in detail.

Let us remark here that the TDA is a popular method in computational chemistry, see e.g., [15]. Because it is formally simpler than the full Casida formalism, it can save computer time, and it has been used in the literature for conceptual analysis of excitation processes (for instance, in the so-called single-pole approximation [16]). Another benefit of the TDA is that there are situations where it is better behaved than the full Casida formalism, for example for open-shell systems away from the ground-state equilibrium geometry, where the TDA avoids so-called triplet instabilities [17].

In this paper, we assess the TDA for TDDFT-LRC exciton binding energies of solids. First, we introduce the various LRC-type kernels to be used in this work and examine the effect of the LRC kernel on excitonic properties of solids. Next, we compare LRC exciton binding energies $E_{\mathrm{b}}^{\mathrm{LRC}}$ of solids obtained from the Casida equation within and beyond the TDA. We discover that the TDA makes very little difference in semiconductors, but has a significant impact in insulators. We discuss the origins, practical implications, and limitations of our findings.

\section{Theoretical Background}

\subsection{Dyson Equation}

In linear-response TDDFT, there are two ways of calculating optical absorption spectra of periodic systems [3]. One way is to use the interacting response function $\chi(\mathbf{q}, \omega)$, which is obtained from the Dyson equation (all quantities are matrices depending on reciprocal lattice vectors $\mathbf{G}, \mathbf{G}^{\prime}$ ):

$$
\chi(\mathbf{q}, \omega)=\chi_{0}(\mathbf{q}, \omega)+\chi_{0}(\mathbf{q}, \omega)\left\{v(\mathbf{q})+f_{\mathrm{xc}}(\mathbf{q}, \omega)\right\} \chi(\mathbf{q}, \omega),
$$

where $v=v_{0}+\bar{v}=4 \pi \delta_{\mathbf{G G}^{\prime}} /|\mathbf{q}+\mathbf{G}|^{2}$ is the Coulomb interaction, and $\chi_{0}$ is the noninteracting response function. $v_{0}$ is the long-range $(\mathbf{G}=0)$ part of the Coulomb interaction, and $\bar{v}$ is the Coulomb interaction without the long-range part. In the optical limit $(\mathbf{q} \rightarrow 0)$, the head $\left(\mathbf{G}=\mathbf{G}^{\prime}=0\right)$ of $\chi_{0}$ is given by [18]

$$
\chi_{0}(\mathbf{q})=-\frac{4 \mathbf{q}^{2}}{(2 \pi)^{3}} \sum_{v c} \int_{\mathrm{BZ}} d \mathbf{k} \frac{\left|\left\langle c \mathbf{k}\left|\hat{p}+i\left[V_{\mathrm{NL}}, \hat{r}\right]\right| v \mathbf{k}\right\rangle\right|^{2}}{\left(E_{c \mathbf{k}}-E_{v \mathbf{k}}\right)^{3}}
$$

where $v$ and $c$ are valence and conduction band indices, respectively, $E_{c, v \mathbf{k}}$ denotes Kohn-Sham single-particle energies, $\hat{p}$ is the momentum operator, $\hat{r}$ is the position operator, and $V_{\mathrm{NL}}$ is the non-local part of the pseudopotential. The optical spectrum is obtained from the macroscopic dielectric function $\epsilon_{\mathrm{M}}$ : 


$$
\begin{aligned}
\epsilon_{\mathrm{M}}(\omega) & =\lim _{\mathbf{q} \rightarrow 0} \frac{1}{\epsilon_{\mathbf{G}=\mathbf{G}^{\prime}=0}^{-1}(\mathbf{q}, \omega)} \\
& =\lim _{\mathbf{q} \rightarrow 0} \frac{1}{1+v_{\mathbf{G}=0}(\mathbf{q}) \chi_{\mathbf{G}=\mathbf{G}^{\prime}=0}(\mathbf{q}, \omega)},
\end{aligned}
$$

where $\epsilon^{-1}$ is the inverse dielectric function. The Dyson-equation approach is computationally relatively cheap, and thus it is the method of choice of most excitonic calculations. However, the method does not allow the precise determination of exciton binding energies, especially if the excitons are weakly bound. The reason is that, in practice, calculations are done with an artificial broadening of several tens of $\mathrm{meV}$, in order to produce spectra that can be compared to experiment. This broadening will completely wash out any excitonic peaks that are on the order of a few tens of meV, which is the case for semiconductors. On the other hand, for insulators, where exciton binding energies are of order $1 \mathrm{eV}$ or more, the peaks are sharp enough to allow exciton binding energies to be read off.

\subsection{Casida Equation}

Alternatively, both optical spectra and exciton binding energies can be obtained from the Casida equation [10]:

$$
\left(\begin{array}{cc}
\mathbf{A} & \mathbf{B} \\
\mathbf{B}^{*} & \mathbf{A}^{*}
\end{array}\right)\left(\begin{array}{l}
X_{n} \\
Y_{n}
\end{array}\right)=\omega_{n}\left(\begin{array}{cc}
-\mathbf{1} & \mathbf{0} \\
\mathbf{0} & \mathbf{1}
\end{array}\right)\left(\begin{array}{l}
X_{n} \\
Y_{n}
\end{array}\right)
$$

where $\mathbf{A}$ and $\mathbf{B}$ are excitation and de-excitation matrices, respectively, $X_{n}$ and $Y_{n}$ are $n$th eigenvectors, and $\omega_{n}$ is the $n$th eigenvalue. The matrix elements of $\mathbf{A}$ and $\mathbf{B}$ are

$$
\begin{aligned}
& A_{v c \mathbf{k}, v^{\prime} c^{\prime} \mathbf{k}^{\prime}}=\left(E_{c \mathbf{k}}-E_{v \mathbf{k}}\right) \delta_{v v^{\prime}} \delta_{c c^{\prime}} \delta_{\mathbf{k} \mathbf{k}^{\prime}}+F_{v c \mathbf{k}, v^{\prime} c^{\prime} \mathbf{k}^{\prime}}^{\mathrm{Hxc}} \\
& B_{v c \mathbf{k}, v^{\prime} c^{\prime} \mathbf{k}^{\prime}}=F_{v c \mathbf{k}, v^{\prime} c^{\prime} \mathbf{k}^{\prime}}^{\mathrm{Hxc}}
\end{aligned}
$$

where $F^{\mathrm{Hxc}}=F^{\mathrm{H}}+F^{\mathrm{xc}}$ is the Hartree-exchange-correlation (Hxc) matrix. In the optical limit, the matrix elements of $F^{\mathrm{Hxc}}$ using the LRC kernel are given by

$$
\begin{aligned}
F_{v c \mathbf{k}, v^{\prime} c^{\prime} \mathbf{k}^{\prime}}^{\mathrm{Hxc}} & =\frac{2}{V}\left(\sum_{\mathbf{G} \neq 0} \frac{4 \pi-\bar{\alpha}}{|\mathbf{G}|^{2}}\left\langle c \mathbf{k}\left|e^{i \mathbf{G} \cdot \mathbf{r}}\right| v \mathbf{k}\right\rangle\left\langle v^{\prime} \mathbf{k}^{\prime}\left|e^{-i \mathbf{G} \cdot \mathbf{r}}\right| c^{\prime} \mathbf{k}^{\prime}\right\rangle\right. \\
& \left.-\alpha_{0} \frac{\left\langle c \mathbf{k}\left|\hat{p}+i\left[V_{\mathrm{NL}}, \hat{r}\right]\right| v \mathbf{k}\right\rangle}{E_{c \mathbf{k}}-E_{v \mathbf{k}}} \frac{\left\langle c^{\prime} \mathbf{k}^{\prime}\left|\hat{p}+i\left[V_{\mathrm{NL}}, \hat{r}\right]\right| v^{\prime} \mathbf{k}^{\prime}\right\rangle^{*}}{E_{c^{\prime} \mathbf{k}^{\prime}}-E_{v^{\prime}} \mathbf{k}^{\prime}}\right) .
\end{aligned}
$$

Here, $V$ is the crystal volume, $\alpha=\alpha_{0} \neq \bar{\alpha}=0$ for $f_{\mathrm{xC}}^{\mathrm{LRC}}=-(\alpha / 4 \pi) v_{0}$ (head-only), and $\alpha=\alpha_{0}=\bar{\alpha} \neq 0$ for $f_{\mathrm{xC}}^{\mathrm{LRC}}=-(\alpha / 4 \pi) v$ (diagonal).

Solving the Casida Equation (5) gives a continuous distribution of energy eigenvalues that lie above the band gap (this is the renormalized single-particle spectrum), and an isolated eigenvalue whose energy is less than the gap (note that this is because we are using a frequency-independent xc kernel, which yields only a single bound exciton [19]). The exciton binding energy is obtained as the difference between the band gap and the lowest, isolated eigenvalue.

Thus, the eigenvalues of the Casida equation yield exciton binding energies in principle with arbitrary precision, in contrast with the Dyson-equation approach, which includes an artificial broadening, as discussed above. In addition, from the eigenvectors of the Casida equations one can obtain oscillator strengths, which can then be used to generate optical spectra. Therefore, the Dyson and Casida approaches are in principle equivalent, but differ in their practical implementation. Furthermore, the Casida approach is computationally significantly more expensive because it requires building and diagonalizing a large matrix. 


\subsection{Local-Field Effect}

The local-field effect (LFE) has different meanings in Dyson and Casida equations. In the Dyson equation, the LFE means that $\epsilon^{-1} \neq 1 / \epsilon$. The Dyson equation is used to calculate optical spectra and Bootstrap-type kernels, which will be explained in Section 4.1. In the Dyson equation for optical spectra, the LFE is not a matter of choice and should be included. However, in the definition of Bootstrap-type kernels, we have the freedom of whether or not to include the LFE, because Bootstrap-type kernels are not constrained by formal derivations. In the following, we chose to include the LFE when calculating Bootstrap-type kernels to be consistent and focus on the TDA.

In the Casida equation, the LFE means that not only the head (i.e., $\mathbf{G}=\mathbf{G}^{\prime}=0$ ) term, but also other terms are included in the summation of $F_{\mathrm{Hxc}}$ matrix elements in Equation (8). In the Casida equation, the LFE is not a matter of choice and should be included.

\subsection{LRC Kernel: Head-Only vs. Diagonal}

Head-only and diagonal LRC kernels, with $f_{\mathrm{xc}}^{\mathrm{LRC}}=-(\alpha / 4 \pi) v_{0}$ and $f_{\mathrm{xc}}^{\mathrm{LRC}}=-(\alpha / 4 \pi) v$, respectively, have been used interchangeably because (i) the form of the LRC kernel is not dictated by a rigorous formal derivation, so the two LRC kernels are largely a matter of choice; (ii) the two LRC kernels cause negligible differences in optical spectra of semiconductors such as $\mathrm{Si}$ (because $\bar{\alpha} \approx 0.2 \ll 4 \pi$ in Equation (8)) [5]. However, as we will report elsewhere [20], we found that the two kernels yield very different results for exciton binding energies of insulators, so it is important to state clearly which version is used. We used the head-only LRC kernel in this work; however, our findings concerning the performance of the TDA hold for both types of LRC kernels. Note that the head-only LRC kernel can be viewed as the diagonal one without the LFE.

\subsection{Tamm-Dancoff Approximation}

The TDA decouples excitations and de-excitations by setting $\mathbf{B}$ to zero in Equation (5). The TDA is widely used in the BSE and the Casida equation because it cuts the computational cost significantly by reducing the size of the exciton Hamiltonian matrix by a factor of two and changing a non-Hermitian eigenvalue problem to a Hermitian one. However, it turns out that the full Casida equation can be solved at the same computational cost as the TDA one [13] using a transformation that is well known from computational chemistry [10]. Making use of time-reversal symmetry, Equation (5) can be transformed to a Hermitian eigenvalue equation:

$$
\mathbf{C} Z_{n}=\omega_{n}^{2} Z_{n}
$$

where

$$
\begin{aligned}
& \mathbf{C}=(\mathbf{A}-\mathbf{B})^{1 / 2}(\mathbf{A}+\mathbf{B})(\mathbf{A}-\mathbf{B})^{1 / 2}, \\
& Z_{n}=(\mathbf{A}-\mathbf{B})^{1 / 2}\left(X_{n}-Y_{n}\right) .
\end{aligned}
$$

\subsection{Band-Gap Corrections: LDA vs. Scissors Shift}

A standard method of producing band structures with the correct band gap is to use so-called scissors operators. There are many ways of applying the scissors shift to Dyson and Casida equations in Equations (3) and (8) and LRC-type kernels. The scissors shift can be applied to only conduction bands (i.e., replacing $E_{c \mathbf{k}}$ by $E_{c \mathbf{k}}+\Delta$ ) or to the momentum operator (i.e., replacing $\hat{p}$ by $\left.\left\{\left(E_{c \mathbf{k}}+\Delta-E_{v \mathbf{k}}\right) /\left(E_{c \mathbf{k}}-E_{v \mathbf{k}}\right)\right\} \hat{p}\right)$ as well [21], where $\Delta$ is the difference between experimental (or $G W)$ and DFT bandgaps.

Due to the many choices involved and the high sensitivity of the LRC kernel, the scissors shift can cause some ambiguities (we will address these issues elsewhere in more detail [20]). In this paper our focus is on the performance of the TDA; we wish to avoid any unnecessary distractions and therefore simply work with uncorrected LDA band structures in both Dyson and Casida equations and in the 
construction of all xc kernels. This impacts the exciton binding energies calculated with and without TDA in the same way (both are calculated relative to the LDA gap), so a meaningful assessment of the TDA is possible. On the other hand, to compare optical spectra with experiment, we simply shift them rigidly by the difference between the LDA gap and the experimental band gap.

\section{Computational Details}

We used the Abinit code for norm-conserving pseudopotentials, Kohn-Sham eigenvectors and eigenvalues, and GW bandgaps within the LDA [22]. We wrote our own TDDFT code for calculating exciton binding energies, and used the dp code for optical spectra [23]. We used experimental lattice parameters and align the optical spectra of GaAs and solid Ne with the experimental band gaps.

In the Dyson equation for optical spectra, we used a $16 \times 16 \times 16$ Monkhorst-Pack k-point mesh, 4 valence bands, and 20 conduction bands for GaAs and solid Ne. In the Dyson equation for Bootstrap-type kernels, we used a $20 \times 20 \times 20(20 \times 20 \times 10) \Gamma$-centered k-point mesh, $4(8)$ valence bands, 20 (20) conduction bands, and 59 (73) G vectors for GaAs, $\beta-\mathrm{GaN}, \mathrm{MgO}$, LiF, solid Ar, and solid $\mathrm{Ne}(\alpha-\mathrm{GaN}$ and AlN). In the Casida equation, we used a $28 \times 28 \times 28(16 \times 16 \times 16)\{16 \times 16 \times 8\}$ [8 $\times 8 \times 8$ ] $\Gamma$-centered k-point mesh, 3 (3) \{6\} [3] valence bands, 2 (6) \{9\} [24] conduction bands, and 59 (59) $\{73\}$ [59] $\mathrm{G}$ vectors for GaAs $(\beta-\mathrm{GaN}$ and $\mathrm{MgO})\{\alpha-\mathrm{GaN}$ and AlN $\}$ [LiF, solid Ar, and solid Ne]. Convergence was carefully tested throughout.

\section{Results and Discussion}

\subsection{Overview of LRC-Type Kernels}

We begin by listing five static LRC-type kernels (empirical LRC, Bootstrap, 0-Bootstrap, RPA-Bootstrap, and JGM kernels) which were used in this work.

The empirical LRC kernel $\left(\alpha=4.615 \epsilon_{\infty}^{-1}-0.213\right.$, where $\epsilon_{\infty}$ is the high-frequency dielectric constant) is the first LRC-type kernel for optical spectra of semiconductors [5]. Note that we used the calculated $\epsilon_{\mathrm{RPA}}^{-1}$ instead of experimental $\epsilon_{\infty}^{-1}$; further, $\epsilon_{\mathrm{RPA}}^{-1}$ is greater than $\epsilon_{\infty}^{-1}$ by $\sim 10 \%$.

The Bootstrap kernel $f_{\mathrm{xc}}^{\text {Boot }}=\epsilon^{-1} / \chi_{0}$, where $\epsilon^{-1}$ is the self-consistent ("bootstrapped") inverse dielectric function and Boot represents Bootstrap, is a parameter-free kernel for optical spectra of semiconductors and insulators [6].

The 0-Bootstrap kernel $\left(f_{\mathrm{xc}}^{0-\text { Boot }}=\epsilon_{\mathrm{RPA}}^{-1} / \chi_{0}\right)$ is the Bootstrap kernel without bootstrapping (i.e., only the first cycle of the self-consistent iteration is carried out). Note that $\alpha_{0-\text { Boot }}$ is greater than $\alpha_{\text {Boot }}$ by $\sim 10 \%$ because $\epsilon_{\text {RPA }}^{-1}$ is greater than $\epsilon^{-1}$ by $\sim 10 \%$.

The RPA-Bootstrap kernel $f_{\mathrm{xC}}^{\mathrm{RPA}-\mathrm{Boot}}=\epsilon_{\mathrm{RPA}}^{-1} / \bar{\chi}_{\mathrm{RPA}}$, where $\bar{\chi}_{\mathrm{RPA}}$ is obtained from $\bar{v}$, is a parameter-free kernel for exciton binding energies of insulators [7]. Note that $\alpha_{\mathrm{RPA}-\mathrm{Boot}}$ is greater than $\alpha_{0-\text { Boot }}$ by $\sim 10 \%$ because $\left|\bar{\chi}_{\text {RPA }}\right|$ is smaller than $\left|\chi_{0}\right|$ by $\sim 10 \%$.

Lastly, the jellium-with-gap-model (JGM) kernel, $\alpha_{\mathrm{JGM}} \approx E_{\mathrm{g}}^{2} / n$, where $E_{\mathrm{g}}$ is the band gap and $n$ is the electron density, is a parameter-free kernel for optical spectra of semiconductors and insulators [9]. Whereas other LRC-type kernels depend on dielectric constants, the JGM kernel depends on band gaps.

We point out again that we used LDA band gaps for all kernels instead of experimental (or GW) band gaps, which affects exciton binding energies of insulators significantly, because our aim is not to test the accuracy of kernels, but to study the effect of the TDA on LRC exciton binding energies. Figure 1 shows the $\alpha$ values of all kernels for different materials. We see that the strength $\alpha$ varies from $\sim 0.1$ ( $\alpha_{\mathrm{RPA}-\text { Boot }}$ for GaAs) to $\sim 30\left(\alpha_{\mathrm{RPA}-\text { Boot }}\right.$ for solid Ne). 


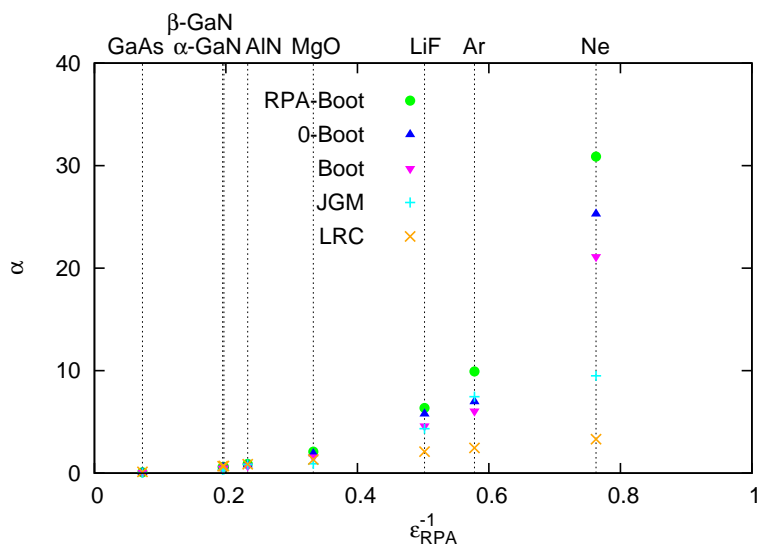

Figure 1. Long-range-corrected (LRC) kernel strengths $\alpha$ (see Equation (1)) of LRC-type kernels for various materials.

\subsection{Effect of the LRC Kernel on Optical Spectra}

Next, we examine the effect of the LRC kernel on optical spectra of solids. Figure 2 shows calculated optical spectra of GaAs and solid Ne obtained from the Dyson equation using $f_{\mathrm{xc}}^{\mathrm{LRC}}=-\alpha / \mathbf{q}^{2}$

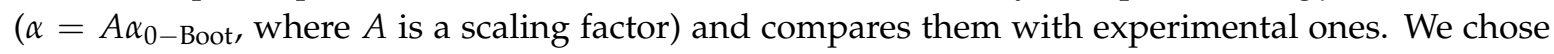
GaAs and solid Ne because they are extreme examples of semiconductors with weakly bound excitons (Wannier-Mott type) and insulators with strongly bound excitons (Frenkel type), respectively.
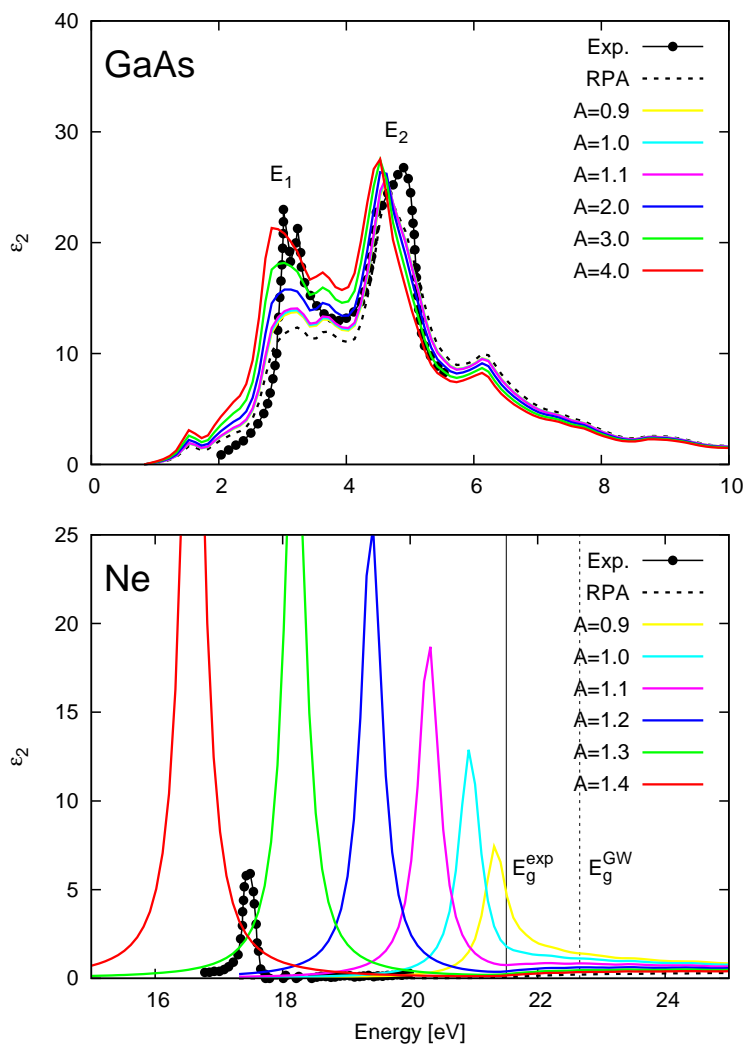

Figure 2. Experimental [24,25] and calculated optical absorption spectra of GaAs (top) and solid Ne (bottom). For the LRC kernel, $\alpha=A \alpha_{0-\text { Boot }}$ is used, where $\alpha_{0-\text { Boot }}=0.064$ (25.3) for GaAs (solid Ne). The spectra are shifted to align the LDA gap with the experimental gap $E_{\mathrm{g}}^{\exp }$; the $G W$ gap $E_{\mathrm{g}}^{G W}$ is shown only for comparison. A Lorentzian broadening of $0.15 \mathrm{eV}(0.2 \mathrm{eV})$ is used for $\mathrm{GaAs}$ (solid Ne). Note that $A=0.9$ and 1.1 approximately correspond to Bootstrap and RPA-Bootstrap kernels, respectively. 
There are important differences between semiconductors and insulators. First, exciton binding energies cannot be easily read off of the optical spectra of semiconductors since the exciton peaks are too close to the gap, and the binding energies tend to be smaller than the spectral broadening; by contrast, the binding energies can be quite accurately obtained from the spacings between experimental gaps and excitonic peaks in the optical spectra of insulators.

Second, LRC spectra of semiconductors are insensitive to $\alpha$ (e.g., a 10\% change in $\alpha$ has little effect on the LRC spectrum of GaAs), whereas LRC spectra of insulators are highly sensitive to $\alpha$ (e.g., a $10 \%$ change in $\alpha$ shifts excitonic peaks by $\sim 1 \mathrm{eV}$ in the spectrum of solid $\mathrm{Ne}$ ). These different effects of the LRC kernel on optical spectra of semiconductors and insulators are important because they are related to different effects of the TDA on LRC exciton binding energies of semiconductors and insulators, which will be shown later. Note that we neglected the effect of the LRC kernel on oscillator strengths or spectral weights (i.e., excitonic peak heights and widths) to focus on excitonic peak positions. We also point out that, whereas we focus here on the two materials GaAs and solid Ne, we have confirmed the generality of our conclusions for many other materials with various degrees of exciton binding strength (see Table 1).

\subsection{TDA and Exciton Binding Energies}

Next, we explore the effect of the TDA on exciton binding energies. Table 1 shows exciton binding energies of different materials obtained from the full and TDA Casida equation using LRC-type kernels. The calculated binding energies are significantly below the experimental results. This is typical for the performance of the different LRC kernels; in a forthcoming publication, we shall analyze this in detail and propose a new LRC kernel which agrees well with experiment [20]. In the present study, our aim is to reveal the differences between the TDA and the full calculation as clearly as possible, rather than reproducing experimental data; therefore, we chose to limit our analysis to the existing unoptimized LRC kernels, and to use LDA gaps instead of scissors corrected gaps.

Table 1. Calculated exciton binding energies (in meV) obtained from Tamm-Dancoff approximation (TDA) and full Casida equations. Experimental exciton binding energies (in meV) are taken from Refs. [26-33] and shown only for comparison.

\begin{tabular}{cccccccccc}
\hline & Casida Equation & GaAs & $\alpha$-GaN & $\beta$-GaN & AlN & MgO & LiF & Ar & Ne \\
\hline Exp. & & $\mathbf{3 . 2 7}$ & $\mathbf{2 0 . 4}$ & $\mathbf{2 6 . 0}$ & $\mathbf{4 8 . 0}$ & $\mathbf{8 0 . 0}$ & $\mathbf{1 6 0 0}$ & $\mathbf{1 9 0 0}$ & $\mathbf{4 0 8 0}$ \\
\hline RPA-Boot & TDA & 0.334 & 0.927 & 0.875 & 0.00 & 1.72 & 33.3 & 37.7 & 666 \\
0-Boot & TDA & 0.285 & 0.811 & 0.720 & 0.00 & 1.43 & 22.4 & 10.8 & 128 \\
Boot & TDA & 0.267 & 0.651 & 0.562 & 0.00 & 1.03 & 10.7 & 7.70 & 39.7 \\
JGM & TDA & 0.137 & 0.387 & 0.226 & 0.00 & 0.348 & 9.12 & 12.9 & 5.30 \\
LRC & TDA & 0.636 & 1.16 & 1.14 & 0.00 & 0.747 & 1.61 & 1.46 & 1.01 \\
\hline RPA-Boot & Full & 0.344 & 1.06 & 1.01 & 0.00 & 2.12 & 94.7 & 96.0 & 2400 \\
0-Boot & Full & 0.293 & 0.919 & 0.829 & 0.00 & 1.72 & 43.2 & 13.7 & 612 \\
Boot & Full & 0.278 & 0.735 & 0.649 & 0.00 & 1.20 & 14.8 & 9.14 & 101 \\
JGM & Full & 0.141 & 0.438 & 0.279 & 0.00 & 0.397 & 12.1 & 17.1 & 5.96 \\
LRC & Full & 0.670 & 1.33 & 1.32 & 0.00 & 0.855 & 1.89 & 1.54 & 1.06 \\
\hline
\end{tabular}

We find that the TDA consistently underestimates the exciton binding energies compared to the full calculation. This is consistent with the known fact that the TDA overestimates BSE eigenvalues [14]. Secondly, the magnitude of the $E_{\mathrm{b}}$ underestimation by the TDA is small for semiconductors, but large for insulators. For instance, full and TDA $E_{b}^{\mathrm{LRC}}$ for GaAS differ by $0.034 \mathrm{meV}$ (a 5\% decrease), whereas full and TDA $E_{\mathrm{b}}^{\mathrm{RPA}-\text { Boot }}$ of solid Ne differ by $1734 \mathrm{meV}$ (a $72 \%$ decrease).

There are two possible causes for the large $E_{\mathrm{b}}$ underestimation by the TDA for insulators: (i) large band gaps (e.g., $E_{\mathrm{g}}^{\mathrm{exp}}=1.43$ and $21.5 \mathrm{eV}$ for GaAs and solid Ne, respectively) or (ii) large $\alpha$ values (e.g., $\alpha_{\mathrm{RPA}-\mathrm{Boot}}=0.12$ and 31 for $\mathrm{GaAs}$ and solid Ne, respectively). The large $E_{\mathrm{b}}$ 
underestimation by the TDA for insulators vanishes when small $\alpha$ values are used. For example, full and TDA $E_{\mathrm{b}}^{\mathrm{LRC}}$ of solid Ne differ by $0.05 \mathrm{meV}$ (a $5 \%$ decrease) because $\alpha_{\mathrm{LRC}}=3.3$ for solid Ne. This indicates that the large $E_{\mathrm{b}}$ underestimation by the TDA for insulators is solely due to large $\alpha$ values. The large $E_{\mathrm{b}}$ underestimation by the TDA (i.e., a $\sim 50 \%$ decrease) starts to appear when $\alpha \approx 10$.

The general trend is thus that the TDA performs well as long as $E_{\mathrm{b}}$ is small compared to the gap (as is the case for semiconductors), but fails when $E_{\mathrm{b}}$ becomes comparable to the gap (as is the case for insulators). (Interestingly, this argument can also be used to rationalize the failure of the TDA to describe plasmons in simple metals, where the gap is zero.) These findings are consistent with the original formulation of TDDFT for excitation energies by Petersilka et al. [16], where the dynamical TDDFT correction to the Kohn-Sham single-particle excitation energies is expressed as a Laurent expansion around individual poles in the response function; as long as the correction to the single-particle spectrum is small, the lowest term in this expansion (which is the TDA) will be appropriate.

\subsection{Comparison of Dyson and Full Casida Equations}

Next, we verify our finding above from the Casida equation using the Dyson equation. In principle, Dyson and Casida equations are equivalent, so they should result in the same exciton binding energy when they use the same kernel. Figure 3 shows exciton binding energies of solid Ne from the Dyson equation (i.e., from Figure 2) and the full and TDA Casida equation as a function of scaling factor $A$.

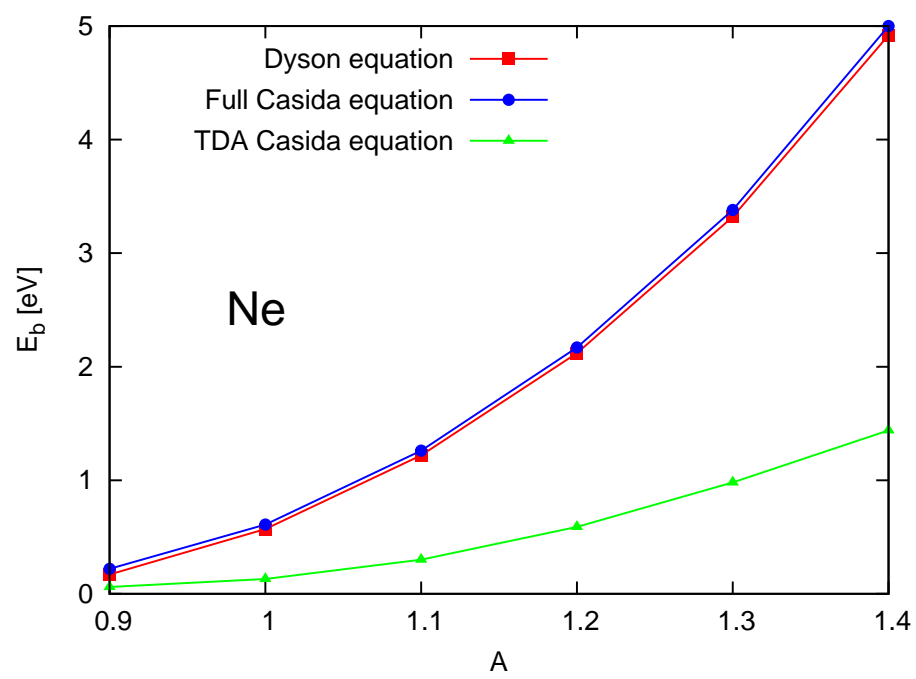

Figure 3. Calculated exciton binding energies $E_{\mathrm{b}}$ of solid $\mathrm{Ne}$ as a function of scaling factor $A$. For the LRC kernel, $\alpha=A \alpha_{0-\text { Boot }}$ is used, where $\alpha_{0-\text { Boot }}=25.3$.

We find at all $A$ values considered that the Dyson and full Casida equations indeed produce almost identical exciton binding energies, and that the TDA underestimates $E_{\mathrm{b}}^{\mathrm{LRC}}$ of solid Ne by a factor of $\sim 3$. This indicates that it is essential to solve the full Casida equation instead of the TDA one when testing whether LRC-type kernels designed for Dyson-equation optical spectra can produce correct and accurate exciton binding energies of insulators.

\subsection{Limitations of Our Findings}

Finally, we discuss the limitations of our findings. First, our conclusions hold only for LRC-type kernels designed for solids. We did not check the effect of the TDA on other types of methods that account for bound excitons in solids (such as the reverse-engineered BSE kernel [2,4], meta-generalized gradient approximations (meta-GGAs) [34] or hybrid xc kernels [12,35-38]) or are designed for atoms and molecules (some discussion of the TDA in the latter case can be found in Ref. [39]). 
The large $E_{\mathrm{b}}$ underestimation by the TDA for insulators is partly due to the high sensitivity of the LRC kernel, which is a unique property of the LRC kernel. Hence, it may not be as pronounced in non-LRC-type kernels.

Secondly, we studied only eigenvalues (i.e., exciton energies), not eigenvectors (i.e., exciton states). The impact of the TDA on oscillator strengths in optical spectra and exciton wavefunctions in real space, which are obtained from eigenvectors, remains to be investigated. Third, we studied only the optical limit ( $\mathbf{q} \rightarrow 0$ ); the effect of the TDA on finite $\mathbf{q}$ values remains to be tested [13]. Lastly, we studied the TDA only for excitons in the optical spectra of bulk materials; in nanoscale systems, additional complications for the TDA can arise [40].

\section{Conclusions}

In summary, we investigated the effect of the TDA on TDDFT-LRC exciton binding energies of solids. We found that the TDA overestimates LRC eigenvalues and thereby underestimates LRC exciton binding energies. This is consistent with the effect of TDA on $E_{\mathrm{b}}^{\mathrm{BSE}}$. We also found that the magnitude of the $E_{\mathrm{b}}^{\mathrm{LRC}}$ underestimation by the TDA depends on the material: it is negligible for semiconductors with small $\alpha$ values, but significant for insulators with large $\alpha$ values. This behavior of the $E_{\mathrm{b}}^{\mathrm{LRC}}$ underestimation by the TDA is similar to that of the $f_{\mathrm{xc}}^{\mathrm{LRC}}$ sensitivity: LRC excitonic properties of semiconductors are rather insensitive to $\alpha$, whereas those of insulators are highly sensitive to $\alpha$.

We quantitatively verified that Dyson and full Casida equations produce identical exciton binding energies. This indicates that it is crucial to solve the full Casida equation instead of the TDA one when studying excitonic properties of insulators using LRC-type kernels.

For now, our conclusions hold only for LRC exciton binding energies of semiconductors and insulators. It will be of interest to study the effect of the TDA for non-LRC-type kernels, and on spectral properties such as oscillator strengths and exciton momentum dispersions.

Acknowledgments: This work was supported by National Science Foundation Grant No. DMR-1408904. The computation for this work was performed on the high performance computing infrastructure provided by Research Computing Support Services at the University of Missouri, Columbia MO.

Author Contributions: Both authors conceived and designed the study; Y.-M.B. performed the numerical calculations; both authors analyzed the data and wrote the paper.

Conflicts of Interest: The authors declare no conflict of interest.

\section{References}

1. Miyata, A.; Mitioglu, A.; Plochocka, P.; Portugall, O.; Wang, J.T.W.; Stranks, S.D.; Snaith, H.J.; Nicholas, R.J. Direct measurement of the exciton binding energy and effective masses for charge carriers in organic-inorganic tri-halide perovskites. Nat. Phys. 2015, 11, 582-587.

2. Onida, G.; Reining, L.; Rubio, A. Electronic excitations: Density-functional versus many-body Green's-function approaches. Rev. Mod. Phys. 2002, 74, 601.

3. Ullrich, C.A.; Yang, Z.H. Excitons in time-dependent density-functional theory. In Density-Functional Methods for Excited States; Ferré, N., Filatov, M., Huix-Rotllant, M., Eds.; Springer: Berlin, Germany, 2015; Volume 368.

4. Reining, L.; Olevano, V.; Rubio, A.; Onida, G. Excitonic effects in solids described by time-dependent density-functional theory. Phys. Rev. Lett. 2002, 88, 066404.

5. Botti, S.; Sottile, F.; Vast, N.; Olevano, V.; Reining, L.; Weissker, H.C.; Rubio, A.; Onida, G.; del Sole, R.; Godby, R.W. Long-range contribution to the exchange-correlation kernel of time-dependent density functional theory. Phys. Rev. B 2004, 69, 155112-155114.

6. Sharma, S.; Dewhurst, J.K.; Sanna, A.; Gross, E.K.U. Bootstrap approximation for the exchange-correlation kernel of time-dependent density-functional theory. Phys. Rev. Lett. 2011, 107, 186401.

7. Rigamonti, S.; Botti, S.; Veniard, V.; Draxl, C.; Reining, L.; Sottile, F. Estimating excitonic effects in the absorption spectra of solids: Problems and insight from a guided iteration scheme. Phys. Rev. Lett. 2015, $114,146402$. 
8. Berger, J.A. Fully parameter-free calculation of optical spectra for insulators, semiconductors, and metals from a simple polarization functional. Phys. Rev. Lett. 2015, 115, 137402.

9. Trevisanutto, P.E.; Terentjevs, A.; Constantin, L.A.; Olevano, V.; Sala, F.D. Optical spectra of solids obtained by time-dependent density functional theory with the jellium-with-gap-model exchange-correlation kernel. Phys. Rev. B 2013, 87, 205143.

10. Casida, M.E. Time-dependent density functional response theory for molecules. In Recent Advances in Density Functional Methods; Chong, D.E., Ed.; World Scientific: Singapore, 1995; Volume 1, pp. 155-192.

11. Yang, Z.H.; Ullrich, C.A. Direct calculation of exciton binding energies with time-dependent density-functional theory. Phys. Rev. B 2013, 87, 195204.

12. Yang, Z.H.; Sottile, F.; Ullrich, C.A. A simple screened exact-exchange approach for excitonic properties in solids. Phys. Rev. B 2015, 92, 035202.

13. Sander, T.; Maggio, E.; Kresse, G. Beyond the Tamm-Dancoff approximation for extended systems using exact diagonalization. Phys. Rev. B 2015, 92, 045209.

14. Shao, M.; da Jornada, F.H.; Yang, C.; Deslippe, J.; Louie, S.G. Structure preserving parallel algorithms for solving the Bethe-Salpeter eigenvalue problem. Linear Algebra Its Appl. 2016, 488, 148-167.

15. Dreuw, A.; Head-Gordon, M. Single-reference ab initio methods for the calculation of excited states of large molecules. Chem. Rev. 2005, 105, 4009-4037.

16. Petersilka, M.; Gossmann, U.J.; Gross, E.K.U. Excitation energies from time-dependent density-functional theory. Phys. Rev. Lett. 1996, 76, 1212-1215.

17. Casida, M.E.; Gutierrez, F.; Guan, J.; Cadea, F.X.; Salahub, D.; Daudey, J.P. Charge-transfer correction for improved time-dependent local density approximation excited-state potential energy curves: Analysis within the two-level model with illustration for $\mathrm{H}_{2}$ and LiH. J. Chem. Phys. 2000, 113, 7062-7071.

18. Baroni, S.; Resta, R. Ab initio calculation of the macroscopic dielectric constant in silicon. Phys. Rev. B 1986, 33, 7017.

19. Yang, Z.H.; Li, Y.; Ullrich, C.A. A minimal model for excitons within time-dependent density-functional theory. J. Chem. Phys. 2012, 137, 014513.

20. Byun, Y.M.; Ullrich, C.A. Systematic assessment of long-range-corrected exchange-correlation kernels for solids: Accurate excitonic properties via a nonuniformly scaled Bootstrap kernel. Phys. Rev. B 2017, In preparation.

21. Levine, Z.H.; Allan, D.C. Linear optical response in silicon and germanium including self-energy effects. Phys. Rev. Lett. 1989, 63, 1719.

22. Gonze, X.; Amadon, B.; Anglade, P.M.; Beuken, J.M.; Bottin, F.; Boulanger, P.; Bruneval, F.; Caliste, D.; Caracas, R.; Côté, M.; et al. ABINIT: First-principles approach to material and nanosystem properties. Comput. Phys. Commun. 2009, 180, 2582-2615.

23. Olevano, V.; Reining, L.; Sottile, F. The Dp Code 1997. Available online: http://www.dp-code.org/ (accessed on 25 January 2017).

24. Lautenschlager, P.; Garriga, M.; Logothetidis, S.; Cardona, M. Interband critical points of GaAs and their temperature dependence. Phys. Rev. B 1987, 35, 9174.

25. Sonntag, B. Dielectric and optical properties. In Rare Gas Solids; Klein, M.L., Venables, J.A., Eds.; Academic Press: London, UK, 1976; Volume II, p. 1021.

26. Parenteau, M.; Carlone, C.; Khanna, S.M. Damage coefficient associated with free exciton lifetime in GaAs irradiated with neutrons and electrons. J. Appl. Phys. 1992, 71, 3747.

27. As, D.J.; Schmilgus, F.; Wang, C.; Schöttker, B.; Schikora, D.; Lischka, K. The near band edge photoluminescence of cubic GaN epilayers. Appl. Phys. Lett. 1997, 70, 1311.

28. Muth, J.F.; Lee, J.H.; Shmagin, I.K.; Kolbas, R.M.; Casey, H.C.; Keller, B.P.; Mishra, U.K.; DenBaars, S.P. Absorption coefficient, energy gap, exciton binding energy, and recombination lifetime of GaN obtained from transmission measurements. Appl. Phys. Lett. 1997, 71, 2572.

29. Haensel, R.; Keitel, G.; Koch, E.E.; Skibowski, M.; Schreiber, P. Reflection spectrum of solid argon in the vacuum ultraviolet. Phys. Rev. Lett. 1969, 23, 1160.

30. Roessler, D.M.; Walker, W.C. Optical constants of magnesium oxide and lithium fluoride in the far ultraviolet. J. Opt. Soc. Am. 1967, 57, 835-836.

31. Saile, V.; Koch, E.E. Bulk and surface excitons in solid neon. Phys. Rev. B 1979, 20, 784. 
32. Leute, R.A.R.; Feneberg, M.; Sauer, R.; Thonke, K.; Thapa, S.B.; Scholz, F.; Taniyasu, Y.; Kasu, M. Photoluminescence of highly excited AlN: Biexcitons and exciton-exciton scattering. Appl. Phys. Lett. 2009, 95, 031903.

33. Roessler, D.M.; Walker, W.C. Electronic Spectrum and Ultraviolet Optical Properties of Crystalline MgO. Phys. Rev. 1967, 159, 733.

34. Nazarov, V.U.; Vignale, G. Optics of semiconductors from meta-generalized-gradient approximation based time-dependent density-functional theory. Phys. Rev. Lett. 2011, 107, 216402.

35. Stephens, P.J.; Devlin, F.J.; Chabalowski, C.F.; Frisch, M.J. Ab initio calculation of vibrational absorption and circular dichroism spectra using density functional force fields. J. Phys. Chem. 1994, 98, 11623.

36. Bernasconi, L.; Tomić, S.; Ferrero, M.; Rérat, M.; Orlando, R.; Dovesi, R.; Harrison, N.M. First-principles optical response of semiconductors and oxide materials. Phys. Rev. B 2011, 83, 195325.

37. Tomić, S.; Bernasconi, L.; Searle, B.G.; Harrison, N.M. Electronic and Optical Structure of Wurtzite CuInS 2 . J. Phys. Chem. C 2014, 118, 14478-14484.

38. Refaely-Abramson, S.; Jain, M.; Sharifzadeh, S.; Neaton, J.B.; Kronik, L. Solid-state optical absorption from optimally tuned time-dependent range-separated hybrid density functional theory. Phys. Rev. B 2015, 92, 081204 .

39. Ullrich, C.A. Time-Dependent Density-Functional Theory: Concepts and Applications; Oxford University Press: Oxford, UK, 2012.

40. Grüning, M.; Marini, A.; Gonze, X. Exciton-plasmon states in nanoscale materials: Breakdown of the Tamm-Dancoff approximation. Nano Lett. 2009, 9, 2820-2824.

(C) 2017 by the authors; licensee MDPI, Basel, Switzerland. This article is an open access article distributed under the terms and conditions of the Creative Commons Attribution (CC BY) license (http:/ / creativecommons.org/licenses/by/4.0/). 